\title{
WebPersona: A Life-Like Presentation Agent for the World-Wide Web
}

\author{
Elisabeth André, Thomas Rist and Jochen Müller \\ German Research Center for Artificial Intelligence (DFKI) \\ Stuhlsatzenhausweg 3, D-66123 Saarbrücken, Germany \\ Email: \{andre,rist,jmueller\}@dfki.de
}

\begin{abstract}
Rapid growth of competition on the electronic market place, will generate the demand for new innovative communication styles with web users. In this paper, we develop an operational approach for the automated generation of hypermedia presentations. Unlike conventional hypermedia, we use a life-like presentation agent which presents the generated material, and guides the user through a dynamically expanding navigation space. The approach relies on a model that combines behavior planning for life-like characters with concepts from hypermedia authoring such as timeline structures and navigation graphs.
\end{abstract}

\section{Introduction}

The World-Wide Web has just begun to change a very broad range of business processes - from marketing and sales, to customer services, order management and distribution. Rapid growth of competition on the electronic market place, will generate the demand for new innovative communication styles with web users. Much effort has already been spent on the conversion of conventional documentation material, such as printed product brochures and instruction manuals, into HTML-hypertext for the distribution on the web.

In the last few years, animated characters based either on cartoon-style drawings [20], real video [11], or geometric 3D-models $[5,21,17,7]$ have become increasingly popular in user interfaces. For web applications, they are a promising option since they make presentations more lively and appealing. They even allow for the emulation of conversation styles common in human-human communication.

Despite of the raging debate on the sociological effects that life-like characters may have, yet can't have, and perhaps never will have, it is safe to say 
that they enrich the repertoire of available options which can be used to effectively communicate information to the user. Among other things, they can be employed to:

- attract the user's focus of attention

- guide the user through a presentation

- realize new presentation means, such as two-handed pointing

- convey additional conversational and emotional signals

With the advent of web-browers which are able to execute programs embedded in webpages, the use of animated characters for the presentation of information over the web has become possible. A web presentation can now comprise dynamic media such as video, animation and speech, all of which have to be displayed in a spatially and temporally coordinated manner. Such a coordination is needed for dynamic presentations in which a life-like character points to and verbally comments on other media objects, such as graphics, video clips, or text passages. The principle is to pack a webpage with:

(1) the media objects along with a specification of how they need to be arranged and temporally scheduled,

(2) a presentation runtime engine which displays the media objects according to the layout specification,

and ship them to the client.

In this paper, we present the PPP Persona (Personalized Plan-based Presenter, [20]), a life-like presentation agent which gathers relevant information from various web sources and data bases, restructures the information into selfcontained units and presents them to the user. Unlike other approaches, e.g., Ball [6], we primarily employ life-like characters for presenting information, and don't support communication with life-like characters via speech. Systems which allow for free-form natural-language input often frustrate users since it is difficult to predict whether a system will be able to handle a request or not. This problem is even aggravated if a life-like character is used because it suggests intelligence. To avoid problems resulting from the deficiencies of current technology for the analysis of spoken language, we offer the user a hypermedia-style interface which allows him to influence the course of a presentation by making specific choices while it is run. The advantage of such an interface is that it makes explicit which kind of interaction possibities the system offers without requiring the user to learn a specific language.

Fig. 1 shows an example. Suppose the user wants to spend holidays in Finland and is looking for a lake-side cottage. To comply with the user's request, the Persona retrieves matching offers from the WWW, selects one of them and presents it to the user. To give the user the possibility of asking for more information, several items in the text are made mouse-sensitive. Clicking on 
one of these items will lead to the insertion of a subscenario. For instance, if the user clicks on the fishing item while the first cottage is presented, the Persona will interrupt the current presentation and run a script with fishing possibilities. After that, it will continue with the main script and describe the next offer. However, following a navigation link does not cause paging as in the case of most conventional web presentations. Rather, a new presentation script for the agent along with the required textual and pictorial material is transferred to the client-side presentation runtime engine.

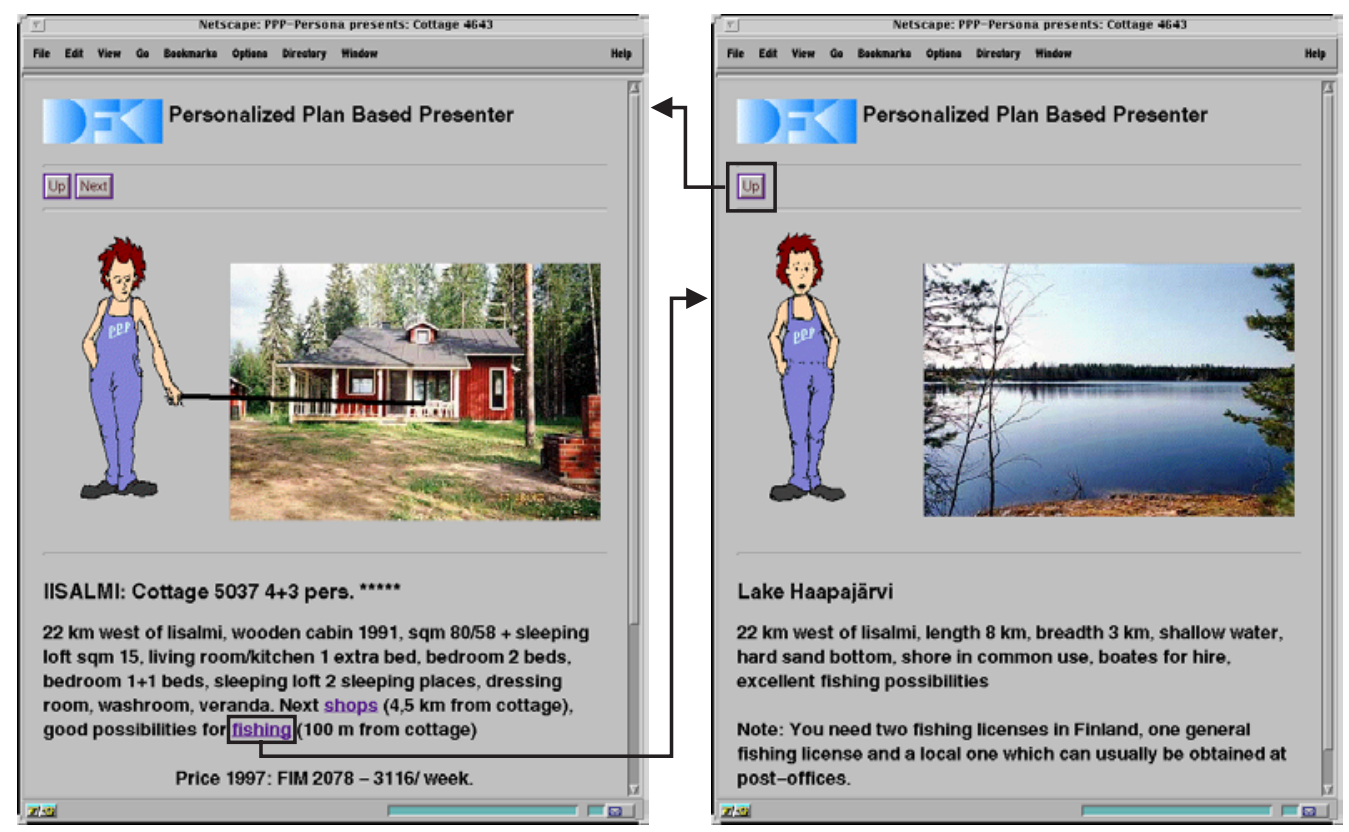

Fig. 1. An Interactive Presentation with the Persona

In the following sections, we describe how to generate such presentations automatically. We first present the underlying knowledge base which maintains both domain and document knowledge. After that, we present a model for interactive hypermedia presentations which combines behavior planning for lifelike characters with concepts from hypermedia authoring. This model forms the basis for our operationalization which will be described in the subsequent two sections. Finally, we report on the outcome of a recent empirical study which compared objective and subjective ratings of presentations with and without a Persona.

\section{Representation of the Underlying Information}

Our system does not store presentation scripts and navigation structures in advance, but generates them automatically from pre-authored document fragments and items stored in a data base. To integrate predesigned and automatically generated material, we start from a hybrid database which comprises 
both information about the domain and information about documents.

Domain information is represented in terms of objects and the relations between them. For example, in the "Cottage-Domain" objects are cottages, lakes, geographic locations, and also activities like hiking, fishing or shopping. A type hierarchy is used to allow for hierarchically structuring domain representations. The set of domain relations may comprise, for example, a part-of relation to express that a certain cottage has a sauna, or a price-relation which may hold between a number and a cottage.

Similar, document information is represented in terms of media objects and relations between them. Media objects are pre-authored document fragments, e.g. a text paragraph or an illustration. Relationships between media objects represent what kind of communicative role a media object may play with respect to another media object in a presentation. For instance, a text paragraph may elaborate on an illustration.

Certainly, media objects serve to present domain information. To bridge the gap between domain information and media objects, we rely on a set of socalled encoding relationships. For example, if the database contains a picture of a certain domain object, then the connection between the two items can be represented by the relation (Depicts pic obj). An encoding relationship is not necessarily a one-to-one mapping. For example, the database may contain several graphics (media objects) for one and the same domain object. Conversely, one and the same media object may be employed for different purposes in different situations.

As Fig. 2 shows, the generation of multimedia presentations can start from information sources which are very different in nature. Note that our approach allows for various degrees of automatization by varying the relative proportion of domain and document knowledge. In the extreme case, the database comprises a very deep domain model from which all media objects can be generated on the fly. We followed this approach in our previous system WIP [2]. Since the current applications of PPP heavily rely on prestored material, a shallow domain model was sufficient. Here, explicit representations of the contents and the structure of the document fragments are required. However, we do not assume completeness of the database in the sense that all possible structural relations are represented. Such an assumption is simply unrealistic for most practical applications. Rather, we follow a principle that states: the more structural relations present in the database, the more the flexibility that can be embodied into the navigation structure of the resulting presentation. Such an approach has also been used for the generation of adaptive hypertext, see e.g. [8]. 

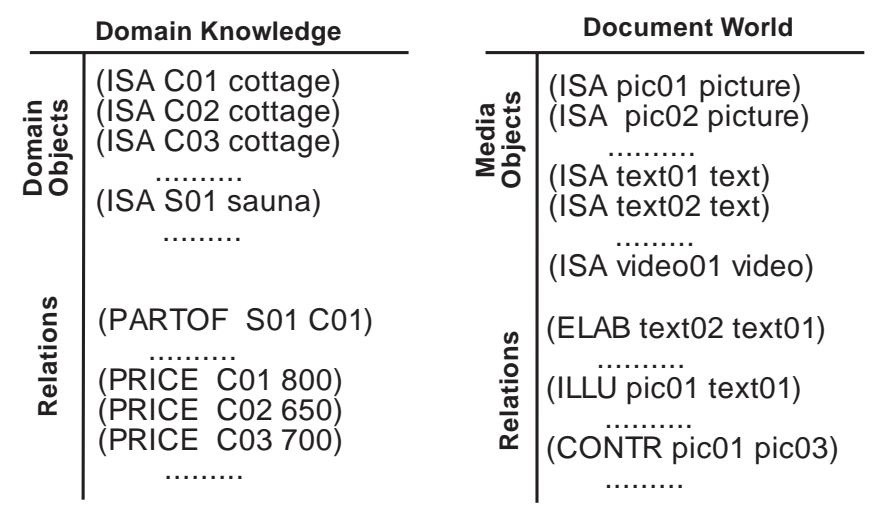

Fig. 2. Representation of the Underlying Information

\section{The WebPersona Presentation Model}

Our presentation model has two main ingrediants: A model which describes the behavior of the character, and a model for the description of hypermedia presentations.

\subsection{The Behavior Model}

What makes up a reasonable behavior for a character depends on a number of factors, such as the chosen metaphor, its purpose, and the conversational setting.

As shown in the example above, our Persona is a cartoon-style human-like figure. Its primary purpose is to execute presentation acts. This includes summarizing the contents of a web page, directing the user's attention to document parts he might be interested in, providing additional information not listed on the web page and recommending links to follow. To accomplish these tasks, the Persona relies on gestures that: express emotions (e.g., approval or disapproval), convey the communicative function of a presentation act (e.g., warn, recommend or dissuade), support referential acts (e.g., to look at an object and point at it), regulate the interaction between the Persona and the user (e.g., establishing eye contact with the user during communication) and indicate that the Persona is speaking. Of course, these gestures may also superimpose each other. For example, to warn the user, the Persona lifts its index finger, looks towards the user and utters the warning.

However, the Persona's behavior is not only determined by the directives (i.e., presentation tasks) specified in the script. Rather, the behavior of the animated character follows the equation: 


\section{Persona behavior $:=$ directives + self-behavior}

Such self-behaviors are indispensible in order to increase the Persona's vividness and believability. Self-behaviors are compiled from different action types (cf. Fig. 3), they currently comprise actions:

\section{- Low-level navigation acts}

In some cases, the Persona has to move to an appropriate position on the screen before carrying out presentation tasks, such as pointing to an object. The kind of navigation act depends on the chosen metaphor. For example, human-like agents like the Persona walk or jump to an appropriate position on the screen while agents like Microsoft's parrot Peedy fly (cf. [7]).

- Idle-time acts

To ensure that the Persona exhibits life-like qualities, it has to stay "alive" even in an idle phase, for instance, when waiting for material to be delivered by the generators or the retrieval components. Typical acts to span pauses are breathing or tapping with a foot.

\section{- Acts that indicate activity}

If the system is still working on a problem, but the character doesn't have to carry out any presentation tasks, it may execute acts to visualize the system's state. For instance, the PPP Persona pulls out a book and starts turning over pages when information is retrieved from the web.

- Reactive behaviors

The Persona should be able to react to user interactions immediately and give visual feedback. For instance, if the user drags the Persona over the screen, the Persona fidgets.

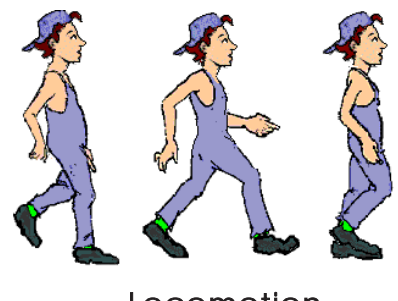

Locomotion

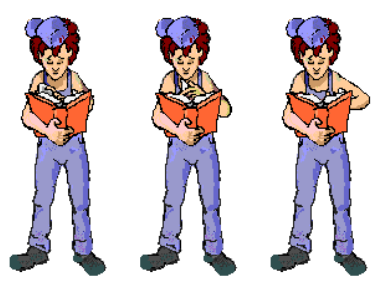

Signa ling Ac tivity
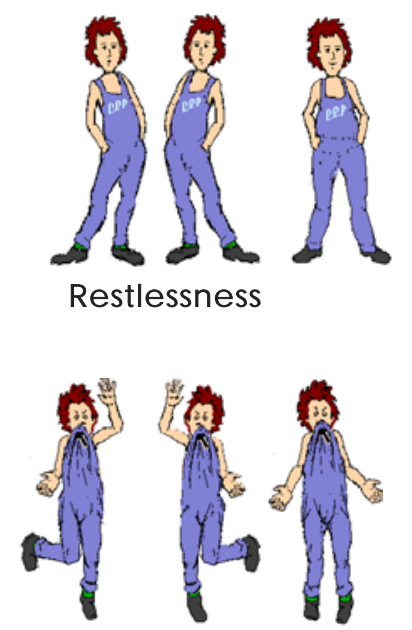

Immediate Reactions

Fig. 3. Classification of Persona Self Behaviors

A distinguishing feature of our model is the clear distinction between taskspecific directives and character- and situation-specific self-behaviors. Though 
it is certainly possible to include appropriate believability-enhancing directly in the presentation script, our approach has an important advantage. From a conceptual point of view, we consider it more adequate since a clear borderline is drawn between a "what to present"- part which is determined by the application, and a "how to present"-part which, to a certain extent, depends on the particular presenter. From the practical perspective, the approach considerably facilitates script production since scripts can be formulated on a higher level of abstraction.

\subsection{The Hypermedia Model}

An important characteristics of our web presentations is that they are not just played back, but have a branching structure which allows the user to choose between different possibilities of navigation. That is, the course of a presentation changes at runtime depending on user interactions. In this section, we will present a model for describing such interactive presentations.

Inspired by the Amsterdam Hypermedia Model [12], we represent web presentation by a collection of presentation units and a set of transitions specifying how to get from one presentation unit to the other.

A presentation unit is defined by a collection of media objects together with a presentation script. We assume that a presentation unit is a self-contained part of a presentation whose media objects are placed in time independent of media objects corresponding to other presentation units.

Presentation scripts entail directions for the character concerning the presentation of media objects. As in other animation scripting systems, we visualize presentation scripts by timeline diagrams which position all actions to be executed by the character along a single time axis. According to the timeline diagram shown in Fig. 4, the Persona first creates window which includes a graphical object, shows it to the user and elaborates on the single parts of the graphical object by pointing to them and providing some additional information verbally. The durations of complex acts correspond to the length of the white bars, the dark bars refer to durations of elementary acts.

Timeline diagrams enable us to represent the temporal behavior of a presentation in an intuitive manner, however, they provide no means of describing the control flow of interactions. Therefore, we combine timeline diagrams with state-transition graphs. That is timeline diagrams are used for describing the temporal behavior of single presentation units while state-transition graphs serve to describe the navigation structure of a presentation.

A state-transition graph $G$ is defined by a set of nodes and edges, i.e. $G$ 
$=\langle N, E\rangle$. With each node $n \in N$, we associate a presentation unit, and a default duration, usually the duration of the presentation unit, i.e. $n:=$ $(<$ duration $><$ presentation_unit $>)$. Each node corresponds to the state of a presentation. If a node is entered, the corresponding presentation script is run. Consequently, being in a certain state means that the corresponding presentation unit is active. An edge $e \in E$ is defined by its connecting nodes, a condition and an action, i.e. $e:=(<$ from $><$ to $><$ condition $><$ action $>)$.
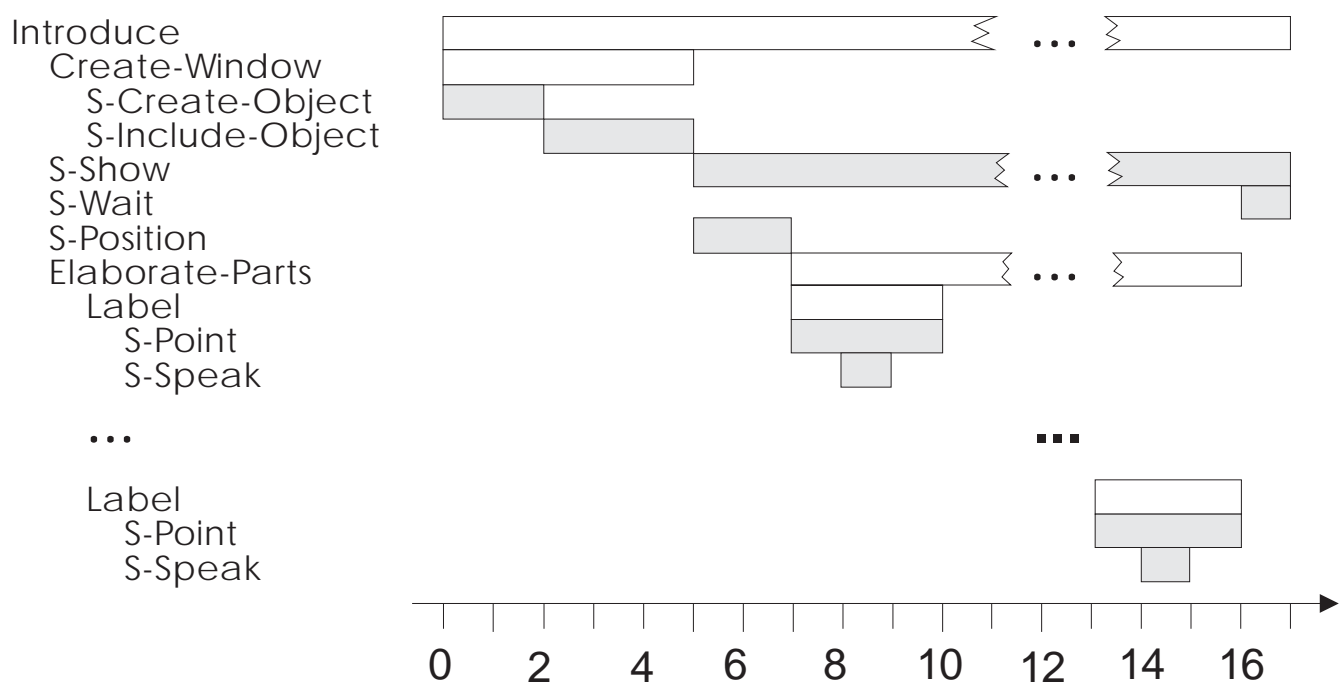

Fig. 4. Example of a Timeline Diagram

A transition is made if one of predicates associated with the edges leading away from the node is satisfied or the default duration is over. Predicates usually refer to user interactions, such as clicking on mouse-sensitive icons in a presentation. An interesting question is the timepoint of transition. Should the system wait until the presentation is completed or interrupt and resume it later? Since a presentation unit may be rather long, we have chosen the second possibility. However, to avoid loosing the coherency of a presentation, we don't allow for the interruption of elementary presentation acts that vary in time, such as speaking or pointing, but wait until these acts are executed. When returning to a node, the system continues the presentation by playing only the remaining part of the script.

Each graph contains a starting and an end node with an empty presentation script and a default duration of 0 . A path through a presentation graph is defined as a sequence of nodes $n_{i}, 1 \leq i \leq m$ where $n_{1}$ is the starting node and $n_{m}$ is the end node. It corresponds to a specific way of viewing the presentation.

The concepts introduced above will be illustrated using the example presented in the introduction. The navigation graph of this example is shown in Fig. 5 . The presentation is started by entering the starting node. Since the default duration of this node is 0 , the first cottage node is entered immediately and 
the corresponding presentation script for the Persona is run. Let's suppose the user clicks on the shopping button while the Persona describes the first cottage. As a consequence, the presentation is interrupted and the shopping script is played. That is the Persona now informs the user about shopping possibilities. After that, the system returns to the first cottage node and plays back the remaining parts of the script. After the default time of 23 time units has passed, a transition is made to the second cottage node. Here, again the user has the possibility of requesting for more information, e.g., about hiking possibilities. After the script for the second cottage has been run and the user hasn't requested for more information, a transition is made to the end node.

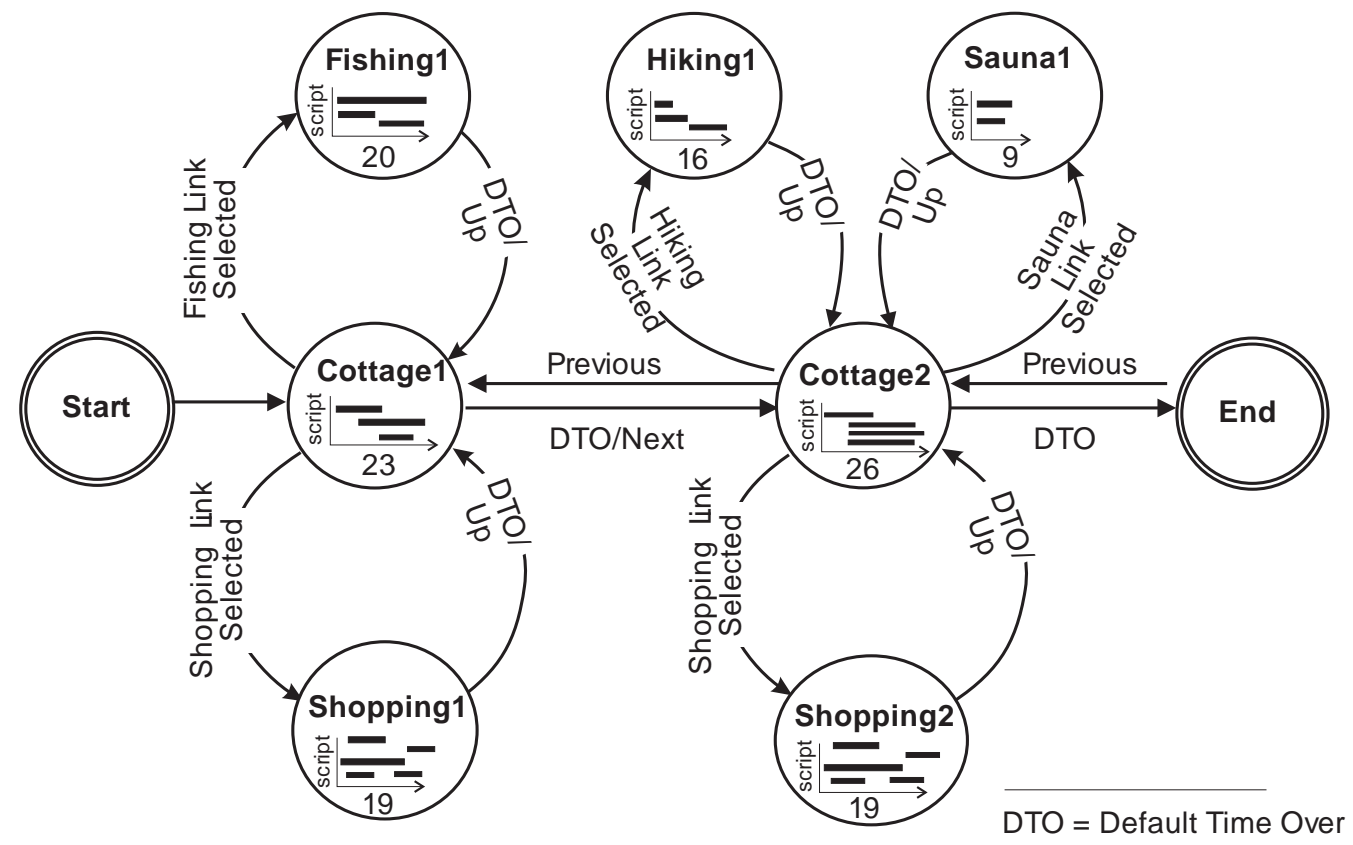

Fig. 5. Navigation Graph for the Cottage Example

\section{Automated Creation of Presentation Scripts}

In the previous section, we presented a model for describing interactive web presentations. However, the manual creation of navigation graphs and presentation scripts is tedious and error-prone. To satisfy the individual needs of a large variety of users, the human author would have to prepare a large number of presentations in advance and to hold them on stock. In the rapidly growing field of online presentation services, the situation is even worse. If live data has to be communicated there is simply not enough time to manually create and continuously update presentations. For example, the nodes of the navigation graph in Fig. 5 correspond to cottages which have been selected for the user on the fly. Since the number of the available cottages and also their features may change at any time, it doesn't make sense to rely on predesigned navi- 
gation graphs or presentation scripts. In the following, we will discuss how to automate the generation process. This process comprises the following tasks:

(1) the design of a multimedia discourse structure reflecting how the single parts of a presentation are related to each other

(2) the decomposition of the presentation into self-contained presentation units

(3) the design of a navigation graph

(4) the design of presentation scripts for each presentation unit

To accomplish these tasks, we build upon our previous work on the automated planning of presentation scripts for presentation agents (cf. [3]), and extend this work for interactive web presentations.

The main idea behind this approach was to formalize action sequences for composing multimedia material and designing scripts for presenting this material to the user as operators of a planning system. The effect of a planning operator refers to a complex communicative goal (e.g. to provide information about a cottage) while the expressions in the body indicate which acts have to be executed in order to achieve this goal (e.g., to show an illustration and to describe it). The temporal behavior of these acts is specified by a list of qualitative and metric constraints. Like other authors in the Multimedia community, e.g. see [18], we represent qualitative constraints in an "Allen-style" fashion (cf. [1]) which allows for the specification of thirteen temporal relationships between two named intervals, e.g. (Speak1 (During) Point2). Quantitative constraints appear as metric (in)equalities, e.g. ( $5 \leq$ Duration Point2).

The input to the presentation planner is a complex presentation goal. To accomplish this goal, the planner looks for operators whose header subsume it. If such an operator is found, all expressions in the body of the operator will be set up as new subgoals. The planning process terminates if all subgoals have been expanded to elementary production/retrieval or presentation tasks or to goals that will be realized by hyperlinks in the final presentation. The result of the planning process is a refinement-style plan which reflects the rhetorical structure of the presentation (see Fig. 6). For example, there is a sequence relationship between the single cottage presentations and elaboration relationships between a cottage presentation and the corresponding subscenarios. Furthermore, this plan specifies how the single parts should be temporally coordinated. For instance, the text and the corresponding illustration should be displayed at the same time.

To allow for the dynamic expansion of the navigation space, we do not expand goals corresponding to hyperlinks at presentation design time, but only if the user selects the corresponding button at presentation runtime. For example, the underlined Elaborate- and Introduce-nodes in Fig. 6 have not yet been 
expanded since the system has decided to realize them as hyperlinks. This method has the advantage that presentations can be adapted to the user's previous navigation behavior and to the information that has been conveyed so far.



Fig. 6. Rhetorical Structure of a Presentation Unit of the Cottage Example

During the planning process, relevant knowledge units for achieving the goals are retrieved from the domain and document knowledge bases and distributed onto different webpages that will be connected by hyperlinks. The presentation of one of these webpages then corresponds to a presentation unit. When creating this network of webpages, the following criteria are considered:

- user characteristics (such as user goals, knowledge and interests)

User characteristics are considered by ranking domain objects and media objects according to their relevance to a particular user or user group. All items of low relevance are realized as hyperlinks. If it's unclear whether an item is of relevance to a particular user, the item becomes a candidate for a hyperlink as well.

- temporal relationships between items

Temporally overlapping presentation parts, such as the acts Illustrate and Emphasize in the cottage example, are assigned to the same presentation unit.

- rhetorical relationships between items

Elaborations are good candidates for hyperlinks, in particular if the information is of lower relevance to the user or space is limited. For instance, all elaborations in the cottage example are realized by separate presentation units because the information is considered less relevant. 
- cohesive links between items

The distribution of material onto different webpages should not disturb the user's viewing process. For example, an illustration should not be separated from its accompanying text if the text contains crossreferences to the illustration. For instance, in the cottage example, the acts S-Display-Text and Illustrate are collected into one presentation unit because there might be crossreferences from text to graphics.

- layout constraints

Items of lower relevance are realized as hyperlinks if the document parts to be presented don't fit on one screen page and scrolling should be avoided.

- optional information

In our approach, the author of plan operators has the possibility to annotate some presentation acts as optional. On the one hand, this method gives the human author more control over the final presentation. On the other hand, the prespecification of hyperlinks reduces the adaptive capabilities of the system at runtime.

For each new presentation unit, the planner creates a node in the navigation graph and specifies how this node can be reached from other nodes and vice versa. These conditions then correspond to the predicates associated with the edges of the navigation graph. For instance, to get from a scenario to an elaborating subscenario, a specific button has to be selected. If the presentation associated with the subscenario is over or the user clicks on an up button, the system returns to the main scenario. To jump back and forth between scenarios connected via a sequence relationship, the user may select a next or previous button resp.

After the rhetorical structure of a presentation unit has been determined, the planner creates a schedule. Since the temporal behavior of each unit can be specified independent of other units, the system can start with this task without knowing which links the user will eventually follow. It first collects all constraints on and between actions of a unit. After that, it determines the transitive closure over all qualitative constraints and computes numeric ranges over interval endpoints and their difference. Finally, a schedule is built up by resolving possibly occurring disjunctions and computing a total temporal order (see [3]). Since the temporal behavior of presentation acts may be unpredictable at design time, the schedule will be refined at runtime by adding new metric constraints to the constraint network.

\section{Transformation of Presentation Scripts into Animation Sequences}

The generated presentation scripts are forwarded to the so-called Persona engine (see Fig. 7) which has to accomplish the following tasks: 
- Determination of the next action to perform

Responses to user interactions usually have the highest priority and may lead to the interruption of a presentation, e.g., if the user signals that he or she is no longer interested in a certain topic. Presentation tasks have a higher priority than idle-time scripts which are only run if the Persona has no other tasks to perform.

- Conversion of high-level presentation tasks into fine-grained animation sequences

This includes the decomposition of high-level presentation tasks into elementary gestures and the satisfaction of necessary preconditions for the execution of these gestures.

- Augmentation of the Persona's behavior by believability-enhancing behaviors In order not to distract the user, idle-time gestures with a high visual impact should only be executed in rare cases. Furthermore, monotonous repetitions should be avoided since they destroy a character's believability.

To support the specification of the Persona's behaviors, we have defined a declarative authoring language which represents Persona behaviors as plan operators. However, while planning techniques have been proven useful for the generation of high-level presentation scripts, animation sequences have to created in realtime and thus require a method which is computationally less expensive. To solve this problem, we follow an approach by [7] and precompile the declarative behavior specifications into a finite state machines. That is we compute for all possible situations beforehand which animation sequence to play. As a result, the system just has to follow the paths of the state machine when making a decision at runtime instead of starting a complex planning process (see [4]).

The compiled state machine forms the so-called behavior monitor (see Figure 7). Besides the behavior monitor, the Persona Engine also comprises an event handler, a character composer, and an interface which is tailored to the target platform (currently either X11 or a Java ${ }^{T M}$ interpreter). The task of the event handler is to recognize whether input derived from the platform interface needs immediate responses from the Persona. That is, for each input message the event handler checks whether the message triggers one of the so-called "reactive behaviors" stored in an internal knowledge-base. If this is the case, the selected behavior is made accessible to the behavior monitor. Depending on the application, notifications may be forwarded to the application program, too. For example in our PPP system, some events are interpreted as requests for the satisfaction of new presentation goals and thus activate a presentation planner (thus the dotted line in Figure 7). The postures determined by the behavior monitor are forwarded to a character composer which selects the corresponding frames (video frames or drawn images) from an indexed database, and forwards the display commands to the window system. 


\section{Application}

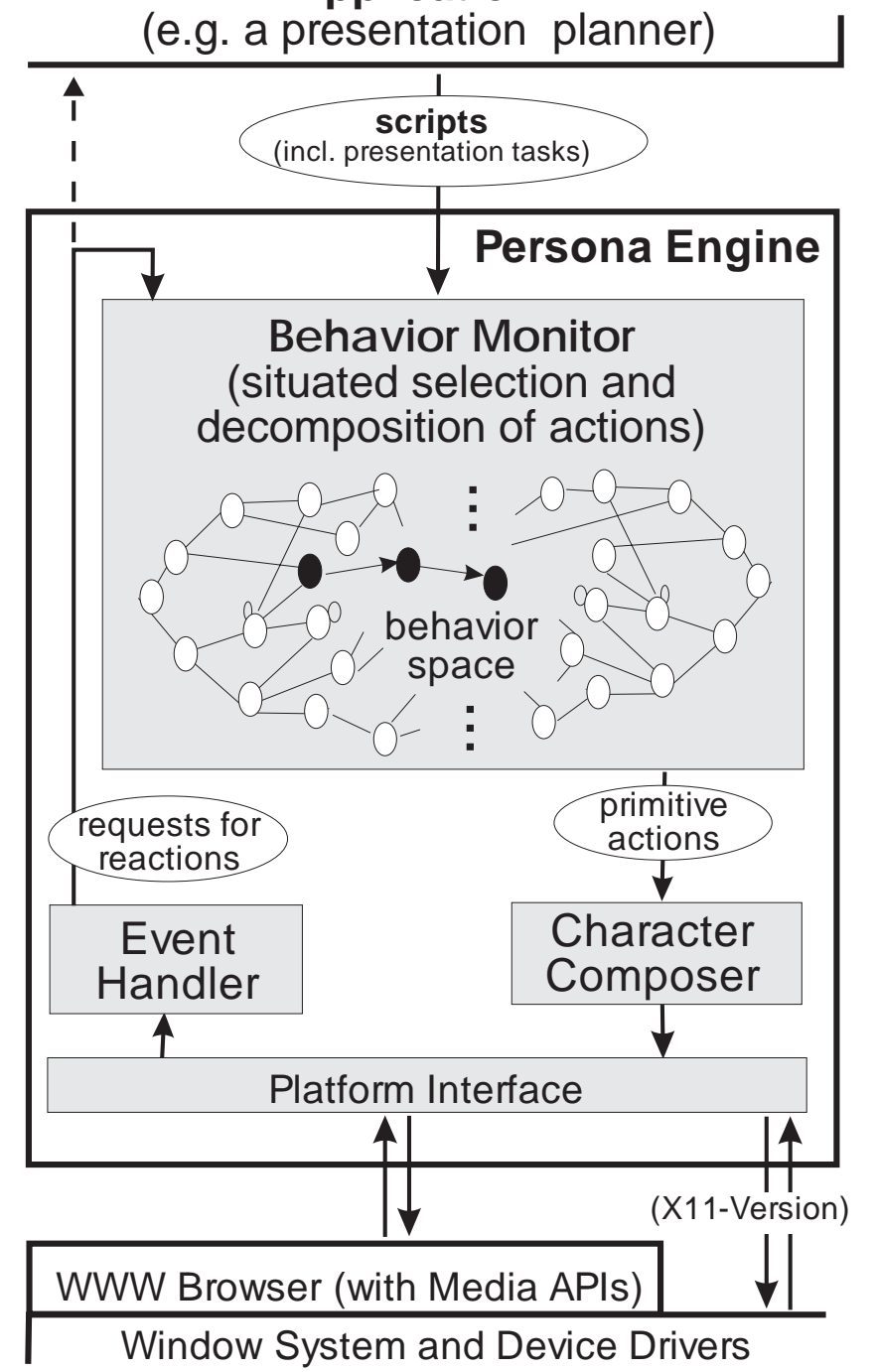

Fig. 7. Architecture of the Persona Engine

\section{Evaluation}

Our research on animated interface agents was motivated by the assumption that they make man-machine communication more effective. In order to find empirical support for this conjecture, we conducted a psychological experiment with 30 adult participants (see also [15]).

Our study focused on two issues: 1) the effect of a Persona on the subject's rating of the presentations (a subjective measure), and 2) his effect on the subject's comprehension of presentations (an objective measure). We thought that three effects on comprehension and recall might occur:

(1) The Persona contributes to the comprehension and recall of presentations 
because of its strong motivational impact.

(2) The Persona has a negative effect on the comprehension and recall of presentations because it distracts the user.

(3) There is no effect of the Persona on comprehension or recall because the Persona neither motivates nor distracts the user or these two factors compensate for each other.

Since earlier studies already provided evidence that a life-like characters has a strong affective impact on the user (e.g., see $[22,24,14]$ ), we expected that this effect also occurs in our case.

\subsection{Experimental Setting}

Participants were 15 females and 15 males, on average 28 years of age, all native speakers of German and recruited from the Saarbrücken university campus. Most of them were no computer specialists, but all of them had some experience in using computers for web surfing and editing purposes.

The subjects were confronted with 5 web-based presentations that they are subsequently asked questions about. Subjects were allowed to spend as much time as they required to answer the questions, but not to watch a presentation several times. On the average, each subject spent 45 minutes on the experiment.

In the experiment, two variables were varied. The first variable referred to the Persona itself. The Persona was either absent or present. In the experiment without the Persona, a voice spoke the same explanations as in the Personaversion and pointing gestures by the Persona were replaced with an arrow. That is the Non-Persona version conveyed exactly the same information as the Persona-version. This was important because we were interested in the effect of the mere presence of a Persona.

The second variable was the information type. Subjects were confronted with technical descriptions of pulley systems and with person descriptions (i.e., information about DFKI employees). In the first case, we showed the subjects illustrations of four different pulley systems and conveyed information concerning the parts of the pulley systems and their kinematics auditorily. Whenever a part of the pulley system was mentioned, a pointing gesture was performed. For the condition with non-technical material, we designed a presentation in which 10 fictitious office employees were introduced. For each employee, his or her photograph was shown and information concerning his or her name and occupation conveyed auditorily. Furthermore, his or her office was pointed at on an office floor layout. 
The first variable was manipulated between-subjects, while the second variable was manipulated within-subjects. Thus, each subject viewed either presentations with or without the Persona, but each subject was confronted with both kinds of presentation. None of the two groups knew about the existence of the other.

The Persona's learning effect was measured by comprehension and recall questions following the presentations. For the technical scenario, the subjects had to answer questions, such as: Which objects does the red rope touch? or "In which direction does the lower pulley move if the free end of the red rope is pulled down?". For the office experiment, we presented the subjects with photographs of office employees and a layout of the office floor. The subjects had to recall the employees' name, occupation and office number.

The Persona's affective impact was measured through a questionnaire at the end of the experiment. Part A of the questionnaire contained general questions on the presentation, such as "Was the presentation difficult to understand?" or "Did you find the presentation entertaining?", while part B contained specific questions on the Persona, such as "How appropriate was the Persona's behavior?", "Did the Persona help you in concentrating on relevant information?" or "Would you prefer presentations with or without a Persona in the future?".

\subsection{Experimental Results}

Regarding our first objective, the evaluation of the Persona's affective impact, our study revealed a positive effect (cf. Tables 1 and 2). Only one subject indicated that he would prefer presentations without a Persona in the future.

T-tests on the data listed in Table 1 show that subjects confronted with technical descriptions ${ }^{1}$ found the subject matter significantly less difficult $(\mathrm{t}(26)=-$ $2.51 ; \mathrm{p}=.0186)$ and the presentation more entertaining $(\mathrm{t}(26)=-2.38 ; \mathrm{p}=.0247)$ in the Persona condition .

In the case of the office experiment, we didn't find a significant difference between the ratings of the difficulty of the presentation and its entertaining value. Also subjects found the Persona's behavior less appropriate in this domain and felt that the Persona was less helpful as a concentration aid (cf. Table 2). We hypothesize that the less positive result for the non-technical domain is due to the fact that the Persona's realization as a workman is more appropriate to technical descriptions than to person-related descriptions.

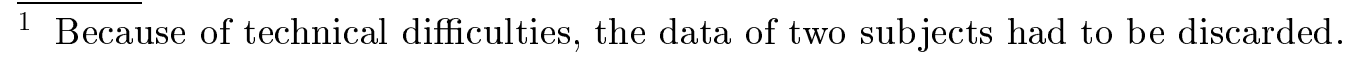


Type of Information

\begin{tabular}{lcccc}
\cline { 2 - 5 } & \multicolumn{2}{c}{ Technical Info } & \multicolumn{2}{c}{ Person Descriptions } \\
\cline { 2 - 5 } Question & Persona Condition & Persona Condition \\
\cline { 2 - 5 } & No Persona & Persona & No Persona & Persona \\
\hline Presentation Difficult & 1.63 & 1.09 & 2.07 & 2.14 \\
Presentation Entertaining & 1.28 & 2.07 & 1.78 & 2.0 \\
Test Difficult & 2.00 & 1.50 & 2.86 & 2.93 \\
Presentation Interesting & 1.71 & 2.21 & 2.0 & 2.28 \\
Information Overload & 1.43 & 1.14 & 2.50 & 2.86 \\
\hline
\end{tabular}

Table 1

Means for the General Questions Asked in the Questionnaire (Part A). Ratings ranging from 0 (negative answer, i.e., indicating disagreement) to 4 (positive answer, i.e., indicating agreement).

\section{Type of Information}

\begin{tabular}{|c|c|c|}
\hline \multirow[b]{2}{*}{ Question } & \\
\hline & Technical Info & Person Descriptions \\
\hline $\begin{array}{l}\text { Persona's behaviour is tuned to pre- } \\
\text { sentation }\end{array}$ & 3.00 & 1.64 \\
\hline $\begin{array}{l}\text { Persona helps concentrate on rele- } \\
\text { vant parts }\end{array}$ & 2.70 & 2.00 \\
\hline $\begin{array}{l}\text { Persona distracts subject from rele- } \\
\text { vant information }\end{array}$ & 1.00 & 0.93 \\
\hline $\begin{array}{l}\text { Persona encourages subject to fur- } \\
\text { ther pay attention to presentation }\end{array}$ & 2.21 & 2.00 \\
\hline
\end{tabular}

Table 2

Means for the Persona-Specific Questions Asked in the Questionnaire (Part B).

Concerning the Persona's learning effect, we didn't find a significant difference between the Persona and the No-Persona version $(\mathrm{t}(26)=-.73 ; \mathrm{p}=.47$ for the technical domain, $\mathrm{t}(26)=.82 ; \mathrm{p}=.42)$ for the non-technical domain). That is the Persona did neither contribute to the students' comprehension of the technical matters in the pulley experiment, nor to the students' recall capabilities in the second experiment (see Table 3).

As a possible reason, we indicate that we only exploited Persona behaviors that can be easily replaced with other means of communication not necessarily requiring the existence of a Persona. In our experiments, Persona gestures were restricted to neutral facial expressions (i.e. head and eye movements towards the objects currently being explained and lip movements indicating that the 
Persona Condition

\begin{tabular}{|c|c|c|}
\hline \multirow[b]{2}{*}{ Type of Info } & \\
\hline & No Persona & Persona \\
\hline Technical Material & 36.14 & 37.57 \\
\hline Person Descriptions & 11.43 & 10.35 \\
\hline
\end{tabular}

Table 3

Means for Comprehension and Recall Performance by the Conditions Persona and Type of Information.

Persona is speaking), pointing gestures and simple idle time actions, such as breathing or tapping with a foot.

On the other hand, initial concerns that people would be distracted by the Persona and concentrate too much on the Persona's facial expressions instead of looking at the referent of the pointing gestures were not confirmed. In the questionnaire, all subjects indicated that the Persona did not distract them (third row of Table 2).

\section{Related Work}

Efforts to the creation of dynamic hypermedia presentations have also been made by the adaptive hypertext and natural-language generation community. As examples, we refer to the ELM-ART [8], ILEX [13] and PEBA-II [9] systems. Like PPP, these systems automatically compose hypertext from canned text and items from a knowledge base. The links between the single pages are automatically created based on the user profile and current situation. However, unlike PPP, they don't allow for the integration of dynamic media, and don't use a life-like character to guide the user through a presentation.

A second line of research which is of interest to our work are approaches to control the behavior of animated interface agents.

Closely related to our work is Microsoft's Persona project in which the interface agent is a parrot named Peedy (cf. [7]). Nevertheless Peedy is an anthropomorphic character since it interacts with the user in a natural-language dialogue, and also mimics some non-verbal (human) communicative acts, e.g., Peedy raises a wing to the ear in case speech recognition fails. Since Peedy is to act as a conversational assistant (at least for the sample application, a retrieval system for music CD's), the system comprises of components for processing spoken language, dialogue management and the generation of audio-visual output. However, the system doesn't have to create presentation scripts since the presentation of material is restricted to playing the selected CDs. 
Lester and Stone [21] have combined a coherence-based behavior sequencing engine to control the behavior of Herman the Bug, the pedagogical agent of Design a Plant. This engine dynamically selects and assembles behaviors from a behavior space consisting of animated segments and audio clips. This material has been manually designed by a multidisciplinary team of graphic artists, animators, musicians and voice specialists. On the one hand, this allows the authoring of high quality presentations as the human author has much control over the material to be presented. On the other hand, enormous effort by the human author is required to produce the basic repertoire of a course. In contrast to their work, our approach aims at a higher degree of automatization. The basic animation units from which a presentation is built correspond to very elementary actions, such as taking a step or lifting one's arm, which are flexibly combined by the Persona Engine. Furthermore, we don't rely on prestored audio clips, but use a speech synthesizer to produce verbal output.

Rickel and Johnson [19] have developed a pedagogical agent called Steve based on the Jack Software, a tool for modeling 3D virtual humans [5]. Instead of creating animation sequences for a course offline and putting them dynamically together as in Design a Plant, the 3D character Steve is directly controlled by commands, such as "look at", walk to" or "grasp an object". In this case, the character interacts with virtual objects in the same way as a human will do in a real environment with direct access to the objects. In contrast to this, our system strictly distinguish between domain and presentation objects. That is the PPP Persona is part of a multimedia presentation and interacts with domain objects via their depictions or descriptions. This setting is similar to a setting where a tutor presents and comments slides or transparencies.

Similar applications have been described by Noma and Badler [16] who developed a virtual human-like presenter based on the Jack Software and Thalmann and Kalra [23] who produced some animation sequences for a virtual character acting as a television presenter. While the production of animation sequences for the TV presenter requires a lot of manual effort, the Jack presenter receives input at a higher level of abstraction. Essentially, this input consists of text to be uttered by the presenter and commands, such as pointing and rejecting, which refer to the presenter's body language. Nevertheless, the human author still has to specifiy the presentation script, while our system computes this automatically starting from a complex presentation goal. However, since our presentation planner is application-independent, it may also be used to generate presentation scripts for the Jack presenter or the TV presenter.

Perlin and Goldberg [17] have developed an "english-style" scripting language called IMPROV for authoring the behavior of animated actors. To a certain extent, the library of agent scripts in their approach can be compared to the repertoire of presentation strategies in our approach since they both allow for the organization of behaviors into groups. However, their scripts are repre- 
sented as a sequence of actions or other scripts while we exploit the full set of Allen relationships. A novelty of our system is that it doesn't require the human author to specify the desired temporal constraints between the single presentation acts, but computes this information dynamically from a complex presentation goal. Furthermore, our system does not only design presentation scripts, but also assembles the multimedia material to be presented to the user.

\section{Technical Data}

The Persona Engine and the Presentation Planner have been implemented in Java $^{T M}$ and $\mathrm{C}++$. While the presentation planner resides on the server side, the Persona Engine is downloaded as a Java ${ }^{T M}$ applet to the user's web brower and receives presentation scripts from the server. Vice-versa, user input can be sent to the presentation server to be considered in the further design process.

The Persona Engine relies on about 250 frames for each Persona. Currently, we use two cartoon personas and three real personas composed of grabbed video material. To control the behavior of the personas, more than 150 different behaviors and about 70 presentation strategies have been defined.

\section{Conclusion}

We have argued that the use of life-like characters are a promising option for presentations on the web. In order to describe such presentations, we combined a behavior model for life-like characters with concepts from hypermedia authoring. Since the manual specification of such presentations would be too labour intensive and error-prone, we also showed how to automate this process. Our current prototype is capable of generating both presentation scripts for life-like characters, and navigation structures to allow the user to dynamically change the course of a presentation at runtime.

While our evaluation study did not support the assumption that life-like agents improve task comprehension and information recall cababilities of human presentation consumers, it clearly revealed a strong affective impact. Our subjects rated learning tasks presented by the Persona as less difficult than presentations without a life-like character. Obviously however, this effect does not occur in all applications, and users seem to have clear preferences about when to have a personified agent in the interface. Thus, user interface designers should not only take into account inter-individual, but also intra-individual differences. 
So far, the focus of our work has been on presentation design. Clearly, the overall quality of the Persona's presentations does not depend solely on its appearance and skills, but to a large extent also on the information gathered from the web. Unfortunately, a presentation agent cannot anticipate which information will be available on the web, i.e. it also has to operate in unknown environments. There are several approaches to tackle this issue. One direction is to rely on methods for information retrieval and extraction. However, we are still far away from robust approaches capable of analysing arbitrary webpages consisting of heterogeneous media objects, such as text, images and video. Another approach uses so-called annotated environments (see [10]) which provide the knowledge agents need to appropriately perform their tasks. These annotations can be compared to markups of a webpage. Our hope is that with the increasing popularity of agents, a standard for such annotations will be developed which will significantly ease the presentation planning process.

We plan to extend our work on presentation agents in several directions. First of all, we are currently defining strategies for presentations involving more than one agent. This extension allows for different role castings, consider for example two agents discussing the pros and cons of a certain product. A new line of research will be opened up with the dissemination of virtual worlds via the web, as life-like agents and so-called avatars will become the inhabitants of these worlds. While the audio-visual realization of these agents will be facilitated by the emerging VRML 2 standard, our technology may be used to have them perform presentation tasks. Finally, we started with the experimental design of a study to evaluate the Persona's effect on the user's navigation behavior. In particular, we would like to investigate in how far recommendations given by different Personas are followed by the user.

\section{Acknowledgments}

This work has been supported by the BMBF under the contracts ITW 94007 and 9701 0. We would like to thank Peter Rist for drawing the cartoons, H.-J. Profitlich and M. Metzler for the development of the temporal reasoner, Frank Biringer for implementing the Persona Compiler, and Susanne van Mulken for supervising the empirical evaluation. In addition, we are grateful for the comments of the anonymous reviewers.

\section{References}

[1] J. F. Allen. Maintaining Knowledge about Temporal Intervals. Communications of the ACM, 26(11):832-843, 1983. 
[2] E. André, W. Finkler, W. Graf, T. Rist, A. Schauder, and W. Wahlster. WIP: The Automatic Synthesis of Multimodal Presentations. In M. Maybury, editor, Intelligent Multimedia Interfaces, pages 75-93. AAAI Press, 1993.

[3] E. André and T. Rist. Coping with temporal constraints in multimedia presentation planning. In Proc. of AAAI-96, volume 1, pages 142-147, Portland, Oregon, 1996.

[4] E. André, T. Rist, and J. Müller. Integrating Reactive and Scripted Behaviors in a Life-Like Presentation Agent. In Proceedings of theSecond International Conference on Autonomous Agents (Agents '98), pages 261-268, Minneapolis/St. Paul, 1998.

[5] N.I. Badler, C.B. Phillips, and B.L. Webber. Simulating Humans: Computer Graphics, Animation and Control. Oxford University Press, New York, Oxford, 1993.

[6] G. Ball. Dialogue initiative in a web assistant. In Proc. of Life-Like Computer Characters '96, Snowbird, Utah, 1996.

[7] G. Ball, D. Ling, D. Kurlander, J. Miller, D. Pugh, T. Skelly, A. Stankosky, D. Thiel, M. van Dantzich, and T. Wax. Lifelike computer characters: the persona project at microsoft. In J.M. Bradshaw, editor, Software Agents. AAAI/MIT Press, Menlo Park, CA, 1997.

[8] P. Brusilovsky, E. Schwarz, and G. Weber. Elm-art: An intelligent tutoring system on world wide web. In C. Frasson and G. Gauthier and A. Lesgold, editor, Intelligent Tutoring Systems (Lecture Notes in Computer Science, Vol. 1086), pages 261-269, Berlin, New York, Heidelberg, 1996. Springer.

[9] R. Dale and Milosavljevic. Authoring on Demand: Natural Language Generation in Hypermedia Documents. In Proceedings of the First Australian Document Computing Symposium (ADCS'96), pages 20-21, Melbourne, Australia, March 1996.

[10] P. Doyle and B. Hayes-Roth. Agents in Annotated Worlds. In Proceedings of theSecond International Conference on Autonomous Agents (Agents '98), pages 173-180, Minneapolis/St. Paul, 1998.

[11] S. Gibbs, C. Breiteneder, V. de Mey, and M. Papathomas. Video Widgets and Video Actors. In Proc. of the UIST'93 (ACM SIGGRAPH Symp. on User Interface Software and Technology), pages 179-184, Atlanta, GA, U.S.A., 1993.

[12] L. Hardman, D.C.A. Bulterman, and G. van Rossum. The Amsterdam Hypermedia Model: Adding Time and Context to the Dexter Model. Communications of the ACM, 37(2):50-62, 1994.

[13] A. Knott, C. Mellish, J. Oberlander, and M. O'Donnell. Sources of Flexibility in Dynamic Hypertext Generation. In Proceedings of the 8th International Workshop on Natural Language Generation, Sussex, 1996. 
[14] J.C. Lester, S. Converse, S. Kahler, T. Barlow, B. Stone, and R. Bhogal. The persona effect: Affective impact of animated pedagogical agents. In Proceedings of $\mathrm{CHI}^{\prime} 97$, pages 359-366, Atlanta, 1997.

[15] S. Van Mulken, E. André, and J. Müller. The Persona Effect: How Substantial Is It. In Proceedings of HCI'98, Sheffield, UK, 1998. to appear.

[16] T. Noma and N.I. Badler. A Virtual Human Presenter. In Proc. of the IJCAI97 Workshop on Animated Interface Agents: Making them Intelligent, pages 45-51, Nagoya, 1997.

[17] K. Perlin and A. Goldberg. Improv: A System for Scripting Interactive Actors in Virtual Worlds. Computer Graphics, 28(3), 1996.

[18] Claudio S. Pinhanez, Kenji Mase, and Aaron Bobick. Interval Scripts: a Design Paradigm for Story-Based Interactive Systems. In CHI97 Conference Proc., pages 287-294, Atlanta, GA, Mar. 1997.

[19] J. Rickel and W.L. Johnson. Integrating pedagogical capabilities in a virtual environment agent. In Proceedings of the First International Conference on Autonomous Agents, Marina del Rey, 1997.

[20] T. Rist, E. André, and J. Müller. Adding Animated Presentation Agents to the Interface. In J. Moore, E. Edmonds, and A. Puerta, editors, Proceedings of the 1997 International Conference on Intelligent User Interfaces, pages 79-86, Orlando, Florida, 1997.

[21] B.A. Stone and J.C. Lester. Dynamically sequencing an animated pedagogical agent. In Proc. of AAAI-96, volume 1, pages 424-431, Portland, Oregon, 1996.

[22] A. Takeuchi and K. Nagao. Communicative facial displays as a new conversational modality. In Proc. of ACM/IFIP INTERCHI'93, pages 187$193,1993$.

[23] N. Magnenat Thalmann and P. Kalra. The Simulation of a Virtual TV presenter. In Computer Graphics and Applications, pages 9-21. World Scientific, 1995.

[24] J.M. Walker, L. Sproull, and R. Subramani. Using a Human Face in an Interface. In Proc. of CHI-94, pages 85-91, Boston, MA, 1994. 